\section{Case Reports in Acute Medicine}

Case Rep Acute Med 2020;3:56-62

\title{
Lightning Injury with Multi-System Involvement
}

\author{
Ashwini Ramesh Patankar Smita Patil Santwana Chandrakar \\ Department of Internal Medicine, Dr. D.Y. Patil Hospital and Medical College, Navi \\ Mumbai, India
}

\section{Keywords}

Lightning injury · Cerebral venous sinus thrombosis · Atrial fibrillation · Sensorineural hearing loss

\section{Abstract}

Lightning leads to trauma and disability such as burns, hearing loss, blindness, cardiac arrhythmia, cardiac arrest, stroke, kidney injury, and can also lead to death. Such cases are seldom reported, and hence their database is scanty. We present a case of a 40-year-old female with lightning injury with four such manifestations, which is not commonly encountered. We have elaborated on these complications of lightning injury and their timely management, which has led us to good patient outcome.

\section{Introduction}

Lightning has been known to cause multiple fatalities. It is also known to cause trauma and disability. Lightning injuries are common in Western India, more in males than females. Lightning injuries have a seasonal predilection to monsoon [1]. These injuries occur more commonly with outdoor activities [2]. Lightning injuries occur not only due to high-voltage electric current, they also occur due to blast waves and temperature changes [3]. The various consequences encountered by lightning injuries are cardiac arrest, arrhythmias, burns, stroke, 
kidney injury, and can lead to death [4]. Occasionally, hearing loss and blindness have been encountered, too [5]. We present a case with lightning injury with its multiple complications and how we managed it.

\section{Case Presentation}

A 40-year-old female farmer was brought to our hospital with complaints of loss of consciousness before arriving at the hospital for a few minutes, vomiting, burns over her neck and abdomen, and decreased hearing after being struck by lightning while working in the farm.

The patient had no known co-morbidities and no cardiac or neurological complaints in the past. Furthermore, she had never been previously evaluated for any disorder, neither cardiac nor neurological.

Upon arrival at the hospital, the patient had severe palpitations and had an irregular heart rate of 180 beats per minute. The patient's blood pressure was 90/50 mm Hg. Rest of the vitals were normal. Her skin over the neck, breast, abdomen, and groin showed second-degree burns ( $36 \%$ by the rule of nines). Immediate electrocardiogram showed atrial fibrillation after which the patient was cardioverted to rate-controlled atrial fibrillation (Fig.1). On systemic examination, heart rate was irregular. Central nervous examination revealed that the patient was conscious, oriented but had bilateral sensorineural hearing loss (SNHL). The patient was then shifted to the intensive care unit for further management.

The patient's basic blood work was normal; however, the cardiac enzymes showed elevations with CK-MB (creatinine kinase), troponin I, and BNP (brain natriuretic peptide) initially. The urine showed presence of protein, blood, and myoglobin. To rule our rhabdomyolysis, total CPK (creatinine phosphokinase) levels were sent. These were within normal limits. Over the duration of admission, the basic blood work remained unremarkable with no dyselectrolytemia or rising creatinine, which is often seen if acute kidney injury occurs. Repeating the urine sample showed normal values, too. The cardiac enzymes after 1 week returned to baseline.

The post-cardioversion electrocardiography showed T-wave inversions in all leads, suggestive of myocardial injury. Echocardiography, however, was normal, although rate-controlled atrial fibrillation persisted.

Upon further investigation by ultrasonography of the abdomen, no internal damage was found. Due to the history of loss of consciousness once the patient was stabilized, magnetic resonance imaging (MRI) of the brain with a venogram was done, which suggested venous sinus thrombosis. An electroencephalogram was also done to rule out seizures as a cause for the loss of consciousness, which showed no abnormal electrical activity. Owing to hearing loss, pure-tone audiometry was done, which confirmed profound sensorineural hearing loss bilaterally (Fig. 2).

The presence of venous thrombosis warranted the need to rule out other hypercoagulable states. Hence, a thrombophilia workup was sent that included antigen and activity of protein C, protein S, and antithrombin III, APCR (activated protein C resistance), lupus anticoagulant, homocysteine, ACL (anticardiolipin antibody) IgG, and ACL IGM. The patient was found to have a protein $\mathrm{C}$ deficiency. Ultrasonography was done to rule out deep vein thrombosis in extremities, and no evidence of it was found.

Primarily, the management of the burns was done with appropriate dressing with silver nitrate $(0.2 \% \mathrm{w} / \mathrm{v})$ application daily. For the atrial fibrillation, oral amiodarone was started which was later tapered, and beta-blockers were started. Due to the cerebral venous 
thrombosis, neurological consult was taken, and after initial low-molecular-weight heparin, oral anticoagulant - warfarin was started with appropriate monitoring of prothrombin time. Intravenous cefotaxime was started in the intensive care unit as prophylaxis for the burns, which was discontinued after 7 days. The patient was shifted to the wards and monitored, and could eventually be discharged.

On follow-up, a repeat brain MRI (venogram) was done (Fig. 3), which did not show significant resolution of the venous thrombosis; hence oral anticoagulant was continued. Betablockers were continued for the atrial fibrillation as the patient continued to have a rate-controlled atrial fibrillation. The site of burns showed good healing with no secondary infection.

For the hearing loss, a repeat pure-tone audiometry was done, which showed improvement in hearing from profound SNHL to moderate SNHL bilaterally.

\section{Discussion and Conclusion}

Lightning injuries have multisystemic manifestations. The cardiac effects of the lightning happen to be a manifest of the direct current of a lightning strike, which can cause cardiac depolarization and asystole [4]. Several reports compiled by Jost et al. [6] have been made of atrial arrhythmias, specifically atrial fibrillation and ventricular arrhythmias following lightning strike; our patient had atrial fibrillation. Usually, these atrial arrhythmias are known to resolve spontaneously, but in our case, the patient being hemodynamically unstable, was cardioverted, yet a rate-controlled atrial fibrillation persisted, which has not been documented so far.

Multiple mechanisms have been proposed by Dronacharya and Poudel [7] to explain the cardiovascular manifestations of lightning injury. These include the induction of coronary artery spasm, catecholamine-mediated effects, direct thermal damage, ischemia secondary to arrhythmia, and coronary artery ischemia.

Burns after electrical injuries are common [8]. Burns can lead to hypotension, as in our patient. It could be postural, cardiac, or secondary to burns (hypovolemic); our patient most likely suffered from either a cardiac cause of hypotension or secondary to burns [9].

Another interesting finding that we had in our patient was bilateral profound SNHL. Usually, the hearing loss is more common secondary to rupture or damage to tympanic membrane, but occasional cases have been reported to have SNHL. This hearing loss is due to either vascular or structural damage to the inner ear [10].

The patient, on neuroimaging, was diagnosed to have a cerebral venous sinus thrombosis. There was no past history suggestive of venous thrombosis, thus this can be a complication of the lightning injury, which has never been encountered and makes this case extremely unique. Van Haren et al. [11] suggest increased hypercoagulable state during recovery from burns, inclusive of electrical burns. This hypercoagulable state could be a cause of cerebral venous thrombosis. The lack of previous case reports on protein $\mathrm{C}$ deficiency suggests the need to further investigate if such cases are encountered.

\section{Statement of Ethics}

The published case report was conducted ethically in accordance with the_World Medical Association Declaration of Helsinki. The patient has given her written informed consent to publish her case (including publication of images). 


\section{Case Reports in Acute Medicine}

\section{Conflict of Interest Statement}

The authors have no conflicts of interest to declare.

\section{Funding Sources}

No funding was required.

\section{Author Contributions}

All authors have contributed to drafting and editing the paper. All authors have given final approval of the version to be published. All authors agree to be accountable for all aspects of the work in ensuring that questions related to the accuracy or integrity of any part of the work are appropriately investigated and resolved.

\section{References}

1 Singh 0, Singh J. Lightning fatalities over India: 1979-2011. Meteorol Appl. 2015;22(4):770-8

2 Apanga PA, Azumah JA, Yiranbon JB. A rare manifestation of burns after lightning strike in rural Ghana: a case report. J Med Case Reports. 2017 Jul;11(1):200.

3 Jones DL, Goyer GG, Plooster MN. Shock wave from a lightning discharge. J Geophys Res. 1968;73(10):31217.

4 McIntyre WF, Simpson CS, Redfearn DP, Abdollah H, Baranchuk A. The lightning heart: a case report and brief review of the cardiovascular complications of lightning injury. Indian Pacing Electrophysiol J. 2010 Sep;10(9):429-34.

5 Faustino LD, Oliveira RA, Oliveira AF, Rodrigues EB, Moraes NS, Ferreira LM. Bilateral maculopathy following electrical burn: case report. Sao Paulo Med J. 2014 Dec;132(6):372-6.

6 Jost WH, Schönrock LM, Cherington M. Autonomic nervous system dysfunction in lightning and electrical injuries. NeuroRehabilitation. 2005;20(1):19-23.

7 Dronacharya L, Poudel R. Lightning induced atrial fibrillation. Kathmandu Univ Med J. 1970;6(4):514-5.

8 Hayashi M, Yamada H, Agatsuma T, Nomura H, Kitahara O. A case of takotsubo-shaped hypokinesis of the left ventricle caused by a lightning strike. Int Heart J. 2005 Sep;46(5):933-8.

9 Latifi NA, Karimi H. Acute electrical injury: A systematic review. J Acute Dis. 2017;6(3):93-6.

10 Turan M, Kalkan F, Bozan N, Özçalimli İ, Zeki Erdem M, Yalınkılıç A, et al. Isolated Sensorineural Hearing Loss as a Sequela after Lightning Strike. Case Rep Otolaryngol. 2015;2015:738416.

11 Van Haren RM, Thorson CM, Valle EJ, Busko AM, Guarch GA, Andrews DM, et al. Hypercoagulability after burn injury. J Trauma Acute Care Surg. 2013 Jul;75(1):37-43. 
Case Reports in Acute Medicine
Case Rep Acute Med 2020;3:56-62

DOI: 10.1159/000509456

(c)

2020 The Author(s). Published by S. Karger AG, Basel

Patankar et al.: Lightning Injury with Multi-System Involvement

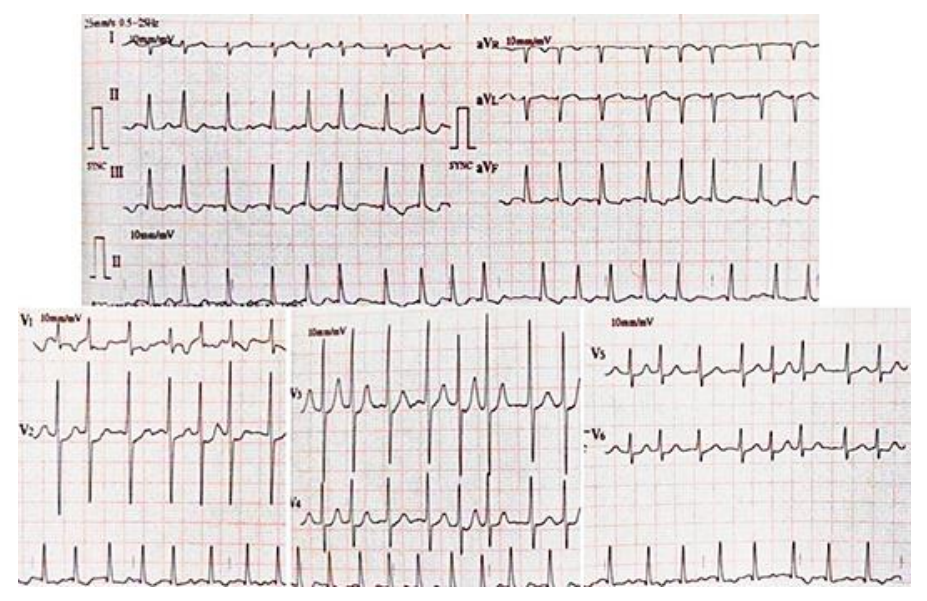

Fig. 1. Electrocardiography before cardioversion. 
Case Reports in Acute Medicine
Case Rep Acute Med 2020;3:56-62

DOI: $10.1159 / 000509456$

2020 The Author(s). Published by S. Karger AG, Basel www.karger.com/cra

Patankar et al.: Lightning Injury with Multi-System Involvement

\section{Frequency in $\mathrm{Hz}$}

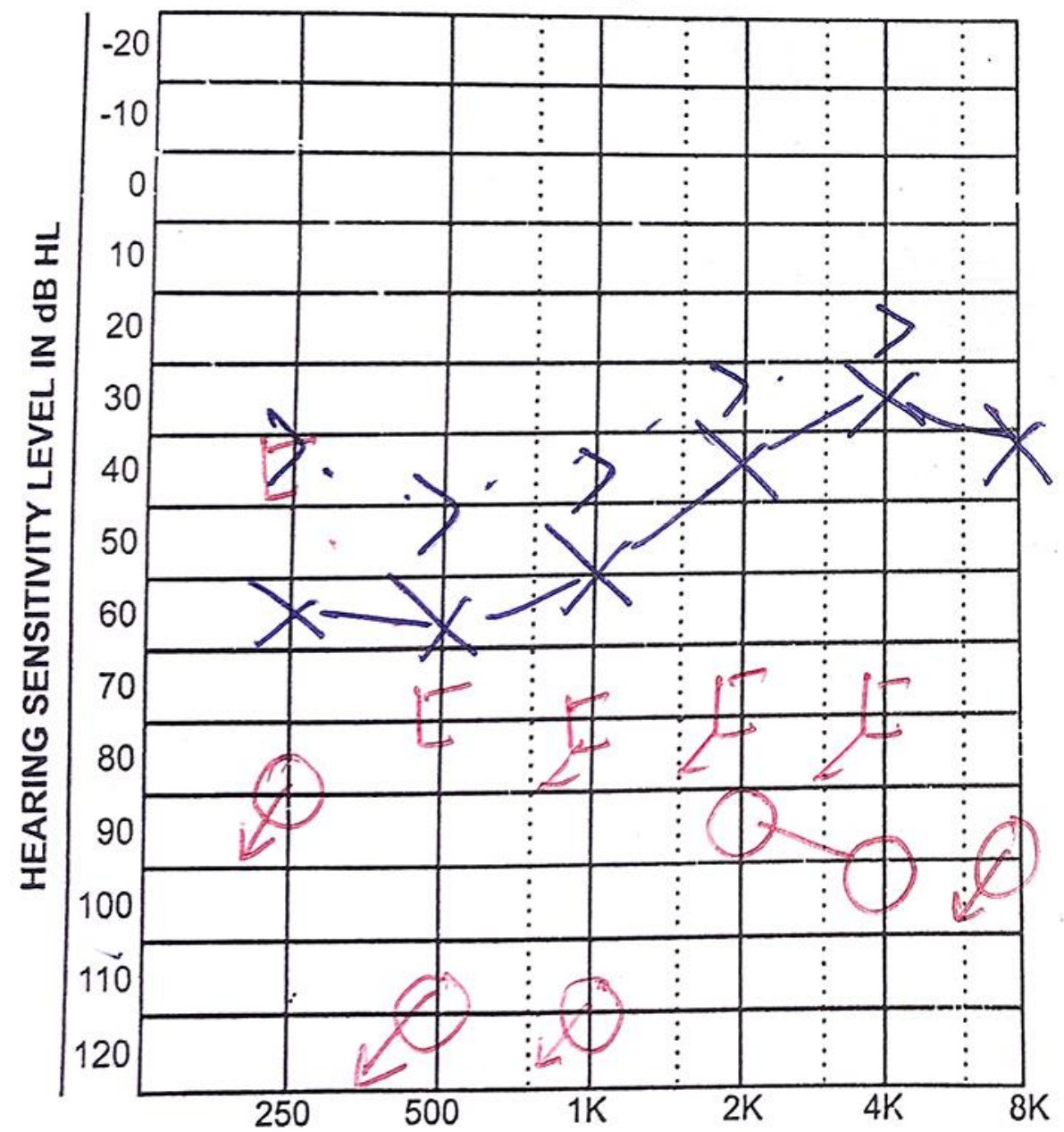

Fig. 2. Pure-tone audiometry of the patient during the first visit. 
Case Reports in Acute Medicine
Case Rep Acute Med 2020;3:56-62

DOI: $10.1159 / 000509456$

The Author(s). Published by S. Karger AG, Basel Patankar et al.: Lightning Injury with Multi-System Involvement

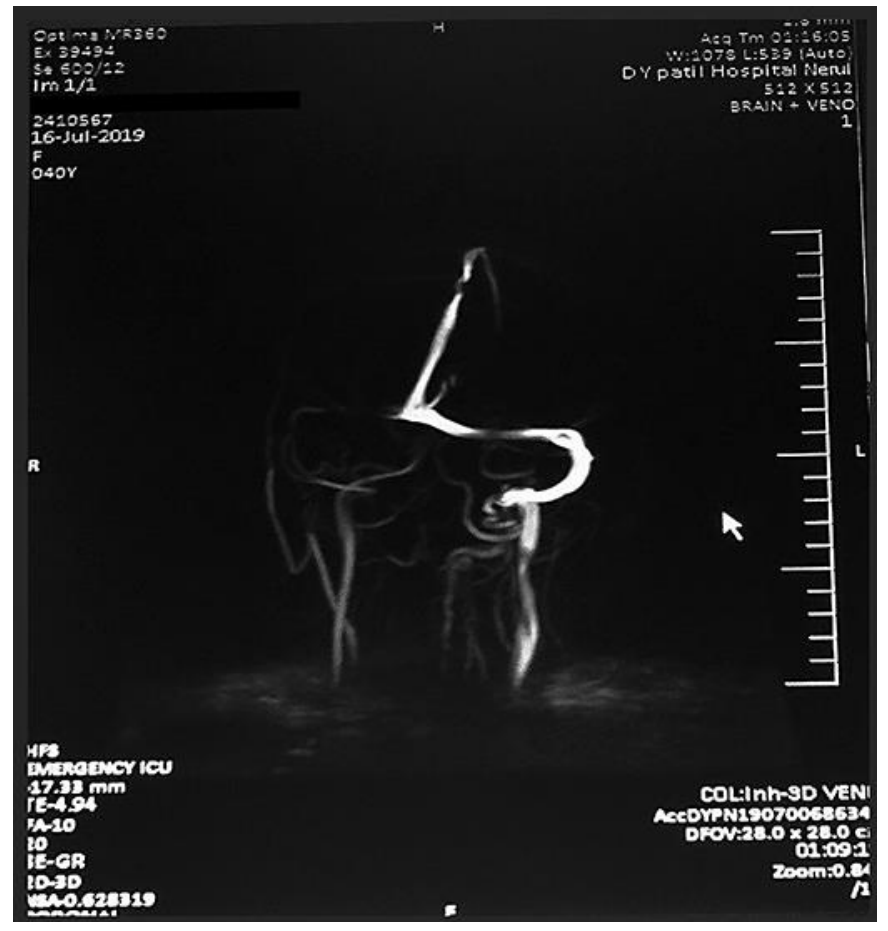

Fig. 3. MRI venogram of the patient. 